\title{
Stochastic Arithmetic as a Model of Granular Computing
}

\author{
René Alt and Jean Vignes
}

\subsection{Introduction}

Numerical simulation is used more and more frequently in the analysis of physical phenomena. A simulation requires several phases.

The first phase consists of constructing a physical model based on the results of experimenting with the phenomena. Next, the physical model is approximated by a mathematical model. Generally, the mathematical model contains algebraic expressions, ordinary or partial differential equations, or other mathematical features which are very complex and cannot be solved analytically. Thus, in the third phase the mathematical model must be transformed into a discrete model which can be solved with numerical methods on a computer. In the final phase the discrete model and the associated numerical methods must be translated into a scientific code by the use of a programming language. Unfortunately, when a code is run on a computer all the computations are performed using floating-point (FP) arithmetic which does not deal with real numbers but with 'machine numbers' consisting of a finite number of significant figures. Thus the arithmetic of the computer is merely an approximation of the exact arithmetic. It no longer respects the fundamental properties of the latter, so that every result provided by the computer always contains a round-off error, which is sometimes such that the result is false. It is therefore essential to validate all computer-generated results. Furthermore, the data used by the scientific code may contain some uncertainties. It is thus also necessary to estimate the influence of data errors on the results provided by the computer.

This chapter is made up in two parts. In the first part, after a brief recalling how round-off error propagation results from FP arithmetic, the CESTAC method (Controle et Estimation STochastique des Arrondis de Calcul) is summarized. This method is a probabilistic approach to the analysis of round-off error propagation and to the analysis of the influence that uncertainties in data have on computed results. It is presented from both a theoretical and a practical point of view. The CESTAC method gives rise to stochastic arithmetic which is presented as a model of granular computing in a similar fashion to interval arithmetic and interval analysis [1]. Theoretically, in stochastic arithmetic the granules are Gaussian random variables and the tools working on these granules are the operators working on Gaussian random variables. In practice, stochastic arithmetic is discretized and is termed discrete stochastic arithmetic (DSA). In this case granules of DSA are the samples provided by the CADNA (Control of Accuracy and

\footnotetext{
Handbook of Granular Computing Edited by Witold Pedrycz, Andrzej Skowron and Vladik Kreinovich
} (C) 2008 John Wiley \& Sons, Ltd 
Debugging for Numerical Application) library which implements the CESTAC method. The construction of these granules and the tools working on them are detailed in this first part.

In the second part, the use of DSA via the CADNA library in three categories of numerical methods is presented. For finite methods, the use of DSA allows the detection of numerical instabilities and provides the number of exact significant digits of the results. For iterative methods the use of DSA allows iterations to be stopped as soon as a satisfactory result is reached and thus provides an optimal (in some sense) termination criterion. Additionally, it also provides the number of exact significant digits in the results. In the case of approximate methods, DSA allows the computation of an optimal step size for the numerical solution of differential equations and the computation of integrals. As in the previous cases, DSA also provides the number of exact significant digits in the results. For each of the three categories, simple but illustrative examples are presented.

\subsection{Round-Off Error Propagation Induced by FP Computation}

A numerical algorithm is an ordered sequence of $v$ operations. For the sake of simplicity it is supposed that the considered algorithm provides a unique result $r \in \mathbb{R}$. When this algorithm is translated into computer and executed, FP arithmetic is used. The obtained result always contains an error resulting from round-off error propagation and is different from the exact result $r$. However, it is possible to estimate this error from the round-off error resulting from each FP operator.

\subsubsection{Errors Due to FP Operators}

Let us consider any value $x \in \mathbb{R}$ in normalized FP form: in this section lowercase letters are used for real numbers and uppercase letters are used for 'machine numbers.' The FP operations on machine numbers are denoted, respectively, by $\oplus, \ominus, \otimes, \oslash$. A real number $x$ is then represented using radix $b$ as

$$
x=\varepsilon \cdot m \cdot b^{e} \quad \text { with } \quad \frac{1}{b} \leq m<1,
$$

where $\varepsilon$ is the sign of $x, m$ is an unlimited mantissa, $b$ is the radix, and $e$ is the integer exponent.

This real number $x$ is represented on a computer working with $b=2$ and a finite length of $p$ bits for the mantissa as $X \in \mathbb{F}, \mathbb{F}$ being the set of FP values which may be represented on a computer and expressed as

$$
X=\varepsilon M \cdot 2^{E},
$$

where $M$ is the limited mantissa encoded using $p$ bits, including the hidden bit, and $E$ is the exponent.

Then, the absolute round-off error resulting from each FP operator is

$$
X-x=\varepsilon M \cdot 2^{E}-\varepsilon \cdot m \cdot b^{e} .
$$

In what follows it is supposed that the two exponents $e$ and $E$ are identical, which is the case most of the time, except, e.g., if $x=1.9999999$ and $X=2.0000000$. So the difference $X-x$, being caused by rounding, is

$$
X-x=\varepsilon 2^{E}(M-m),
$$

with the finite mantissa $M$ and the infinite mantissa $m$ being identical up to the $p$ th bit. Consequently,

- For the assignment operator, this round-off error can be expressed by equation (3):

$$
X=x-\varepsilon \cdot 2^{E-p} \cdot \alpha .
$$

For the rounding to nearest mode: $\alpha \in[-0.5,0.5[$ 
For the rounding to zero mode: $\alpha \in[0,+1[$

For the rounding to $-\infty$ or to $+\infty$ mode: $\alpha \in]-1,+1[$

- For the addition operator $\oplus$

$$
\begin{aligned}
& x_{1} \in \mathbb{R} \quad x_{2} \in \mathbb{R} \quad X_{1} \in \mathbb{F} \quad X_{2} \in \mathbb{F} \\
& X_{i}=x_{i}-\varepsilon_{i} 2^{E_{i}-p} \alpha_{i} \quad i=1,2 \\
& X_{1} \oplus X_{2}=x_{1}+x_{2}-\varepsilon_{1} 2^{E_{1}-p} \alpha_{1}-\varepsilon_{2} 2^{E_{2}-p} \alpha_{2}-\varepsilon_{3} 2^{E_{3}-p} \alpha_{3},
\end{aligned}
$$

where $E_{3}, \varepsilon_{3}$, and $\alpha_{3}$ are, respectively, the exponent, the sign, and the round-off error resulting from the FP addition.

- For the subtraction operator $\ominus$

$$
X_{1} \ominus X_{2}=x_{1}-x_{2}-\varepsilon_{1} 2^{E_{1}-p} \alpha_{1}+\varepsilon_{2} 2^{E_{2}-p} \alpha_{2}-\varepsilon_{3} 2^{E_{3}-p} \alpha_{3} .
$$

- For the multiplication operator $\otimes$

$$
X_{1} \otimes X_{2}=x_{1} x_{2}-\varepsilon_{1} 2^{E_{1}-p} \alpha_{1} x_{2}-\varepsilon_{2} 2^{E_{2}-p} \alpha_{2} x_{1}+\varepsilon_{1} \varepsilon_{2} 2^{E_{1}+E_{2}-2 p} \alpha_{1} \alpha_{2}-\varepsilon_{3} 2^{E_{3}-p} \alpha_{3} .
$$

In equation (7) the fourth term is of second order in $2^{-p}$. When this term is neglected, the approximation to the first order of the round-off error resulting from the FP multiplication is expressed as equation (8):

$$
X_{1} \otimes X_{2} \simeq x_{1} x_{2}-\varepsilon_{1} 2^{E_{1}-p} \alpha_{1} x_{2}-\varepsilon_{2} 2^{E_{2}-p} \alpha_{2} x_{1}-\varepsilon_{3} 2^{E_{3}-p} \alpha_{3}
$$

- For the division operator $\oslash$

In the same way as that for the multiplication, the approximation to the first order of the round-off error is expressed as equation (9):

$$
X_{1} \oslash X_{2} \simeq \frac{x_{1}}{x_{2}}-\varepsilon_{1} 2^{E_{1}-p} \frac{\alpha_{1}}{x_{2}}+\varepsilon_{2} 2^{E_{2}-p} \alpha_{2} \frac{x_{1}}{x_{2}^{2}} .
$$

\subsubsection{Error in a Computed Result}

Starting from the equations in Section 2.2.1, the absolute round-off error on the computed result $R$ of a code requiring $v$ FP operations including assignments can be modeled to first order in $2^{-p}$ by equation. (10)

$$
R=r+\sum_{j=1}^{\nu} g_{j}(d) 2^{-p} \alpha_{j},
$$

where $g_{j}(d)$ are quantities depending exclusively on the data and on the code but independent of the $\alpha_{j}$ 's and $r$ and $R$ are the exact result and the computed result respectively. This formula has been proved in $[2,3]$.

\subsection{The CESTAC Method, a Stochastic Approach for Analyzing Round-Off Error Propagation}

In the stochastic approach the basic idea is that during a run of a code round-off errors may be randomly positive or negative with various absolute values. Thus in equation (10), the coefficients $\alpha_{j}$ 's may be considered as independent random variables. The distribution law of $\alpha_{j}$ 's has been studied by several authors. First, Hamming [4] and Knuth [5] showed that the most realistic distribution of mantissas is 
a logarithmic distribution. Then, on this basis, Feldstein and Goodman [6] proved that the round-off errors denoted by the $\alpha_{j}$ 's can be considered as random variables uniformly distributed on the intervals previously defined in Section 2.2.1 as soon as the number of bits $p$ of the mantissa is greater than 10. Note that in practice $p \geq 24$. In this approach a computed result $R$ can be considered as a random variable, and the accuracy of this result depends on the characteristics of this random variable, i.e., the mean value $\mu$ and the standard deviation $\sigma$. This means that the higher the value of $\frac{\sigma}{\mu}$, the lower the accuracy of $R$. But for estimating $\mu$ and $\sigma$ it is necessary to obtain several samples of the distribution of $R$. Unfortunately, during the computation information on round-off errors is lost.

In consequence, how is it possible to obtain several samples of the computed result $R$ ? The CESTAC method gives an easy answer to this question.

\subsubsection{Basic Ideas of the Method}

The CESTAC method was first developed by M. La Porte and J. Vignes [7-11] and was later generalized by the later in [12-19].

The basic idea of the method is to execute the same code $N$ times in a synchronous manner so that round-off error propagation is different each time.

In doing this $N$ samples are obtained using a random rounding mode.

\subsubsection{The Random Rounding Mode}

The idea of the random rounding mode is that each result $R \in \mathbb{F}$ of an FP operator (assignment, arithmetic operator), which is not an exact FP value, is always bounded by two FP values $R^{-}$and $R^{+}$obtained, respectively, by rounding down and rounding up, each of them being representative of the exact result. The random rounding consists, for each FP operation or assignment, in choosing the result randomly and with an equal probability, as $R^{-}$or $R^{+}$.

When a code is performed $N$ times in a synchronous parallel way using this random rounding mode, $N$ samples $R_{k}, k=1, \ldots, N$, are obtained of each computed result. From these samples, the accuracy of the mean value $\bar{R}$, considered to be the computed result, may be estimated as explained in the following section.

\subsubsection{Modeling the CESTAC Method}

From the formalization of the round-off error of the FP operators presented in Section 2.2, a probabilistic model of the round-off error on a computed result obtained with the random rounding mode has been proposed. This model is based on two hypotheses:

- Hyp. 1: The elementary round-off errors $\alpha_{j}$ of the FP operators are random, independent, uniformly distributed variables.

- Hyp. 2: The approximation of the first order in $2^{-p}$ is legitimate.

Hypothesis 2 means that the terms in $2^{-2 p}$, which appear in the expression of the round-off error of FP multiplications and FP divisions, have been neglected. Only the terms in $2^{-p}$ are considered.

It has been shown $[2,3]$ that if the two previous hypotheses hold, each sample $R_{k}$ obtained by the CESTAC method may be modeled by a random variable $Z$ defined by

$$
\left.Z \simeq r+\sum_{i=1}^{v} u_{i}(d) 2^{-p} z_{i} \quad z_{i} \in\right]-1,+1[,
$$

where $u_{i}(d)$ are constants, $v$ is the number of arithmetic operations, and $z_{i}$ are the relative round-off errors on mantissas considered as independent, centered, and equidistributed variables. 
Then

- $\bar{R}=E(Z) \simeq r$.

- The distribution of $Z$ is a quasi-Gaussian distribution. Consequently to estimate the accuracy of $\bar{R}$ it is legitimate to use Student's test which provides a confidence interval for $\bar{R}$ and then to deduce from this interval the number of significant decimal digits $C_{\bar{R}}$ of $\bar{R}$, which is estimated by equation (12).

$$
C_{\bar{R}}=\log _{10}\left(\frac{\sqrt{N}|\bar{R}|}{\sigma \tau_{\eta}}\right)
$$

where

$$
\bar{R}=\frac{1}{N} \quad \sum_{k=1}^{N} R_{k} \quad \text { and } \quad \sigma^{2}=\frac{1}{N-1} \sum_{k=1}^{N}\left(R_{k}-\bar{R}\right)^{2},
$$

$\tau_{\eta}$ is value of Student's distribution for $N-1$ degrees of freedom and a probability level $1-\eta$.

Remark. The statistical property used here is the following. Let $m$ be the unknown mean value of a Gaussian distribution. Then, if $\left(x_{k}\right), k=1, \ldots, N$, are $N$ measured values satisfying this distribution, $\bar{x}$ is the mean value of the $x_{k}$, and $\sigma$ is the empirical standard deviation defined as $\sigma=\sqrt{\frac{1}{N-1} \sum_{k=1}^{N}\left(x_{k}-\bar{x}\right)^{2}}$, the variable $T=\frac{\bar{x}-m}{\sigma} \sqrt{N}$ satisfies a Student's distribution with $N-1$ degrees of freedom. For example for $N=3$ and $1-\eta=0.05$, i.e., a percentile of 0.975 , Student's table for $N-1=2$ degrees of freedom provides a value $\tau_{\eta}=4.303$.

From a theoretical point of view, we may define a new number called a 'stochastic number,' which is a Gaussian variable defined by its mean value and its standard deviation. The corresponding operators for addition, subtraction, multiplication, and division define what is called stochastic arithmetic.

\subsection{Stochastic Arithmetic}

Stochastic arithmetic operates on stochastic numbers and is directly derived from operations on independent Gaussian random variables. Let us present here the main properties of this arithmetic, which are detailed in $[20,21]$.

From the granular computing point of view a stochastic number is a granule and the stochastic arithmetic is a tool for computing granules.

\subsubsection{Definition of the Stochastic Operators}

Definition 1. Stochastic numbers (granules).

The set of stochastic numbers denoted $\mathbb{S}$ is the set of Gaussian random variables. Then an element $X \in \mathbb{S}$ is defined by $X=(m, \sigma), m$ being the mean value of $X$ and $\sigma$ being its standard deviation.

If $X \in \mathbb{S}$ and $X=(m, \sigma)$, then $\lambda_{\eta}$ exists (depending only on $\eta$ ) such that

$$
\begin{array}{r}
P\left(X \in\left[I_{\lambda, X}\right]\right)=1-\eta \\
I_{\eta, X}=\left[m-\lambda_{\eta} \sigma, m+\lambda_{\eta} \sigma\right] .
\end{array}
$$

$I_{\eta, X}$ is the confidence interval of $m$ with a probability $(1-\eta)$. For $\eta=0.05, \lambda_{\eta}=1.96$. Then the number of significant digits on $m$ is obtained by

$$
C_{\eta, X}=\log _{10}\left(\frac{|m|}{\lambda_{\eta} \sigma}\right)
$$


Definition 2. Stochastic zero.

$X \in \mathbb{S}$ is a stochastic zero, denoted $\underline{0}$, if and only if

$$
C_{\eta, X} \leq 0 \quad \text { or } \quad \mathrm{X}=(0,0) .
$$

Note that if mathematically $f(x)=0$, then with stochastic arithmetic $F(X)=\underline{0}$.

Definition 3. Stochastic operators (tools working on granules).

The four elementary operations of stochastic arithmetic between two stochastic numbers $X_{1}=$ $\left(m_{1}, \sigma_{1}\right)$ and $X_{2}=\left(m_{2}, \sigma_{2}\right)$, denoted by $s+, s-,{ }_{s} \times, s /$, are defined by

$$
\begin{aligned}
& X_{1} \quad \text { st } \quad X_{2} \stackrel{\text { def }}{=}\left(m_{1}+m_{2}, \sqrt{\sigma_{1}^{2}+\sigma_{2}^{2}}\right), \\
& X_{1} \quad \text { s- } \quad X_{2} \stackrel{\text { def }}{=}\left(m_{1}-m_{2}, \sqrt{\sigma_{1}^{2}+\sigma_{2}^{2}}\right), \\
& X_{1} \quad \text { s } \quad X_{2} \stackrel{\text { def }}{=}\left(m_{1} m_{2}, \sqrt{m_{2}^{2} \sigma_{1}^{2}+m_{1}^{2} \sigma_{2}^{2}+\sigma_{1}^{2} \sigma_{2}^{2}}\right), \\
& X_{1} \quad \text { s } \quad X_{2} \stackrel{\text { def }}{=}\left(m_{1} / m_{2}, \sqrt{\left(\frac{\sigma_{1}}{m_{2}}\right)^{2}+\left(\frac{m_{1} \sigma_{2}}{m_{2}}\right)^{2}}\right), \quad \text { with } m_{2} \neq 0 .
\end{aligned}
$$

Remark. The first three formulas including stochastic multiplication are exact. However the last one is often simplified by considering only the first-order terms in $\frac{\sigma}{m}$, i.e., by suppressing the term $\sigma_{1}{ }^{2} \sigma_{2}{ }^{2}$ [21], but this is acceptable only when this ratio is small, which is not always the case. The formula for the division is defined only to the first-order terms in $\frac{\sigma}{m}$.

Definition 4. Stochastic equality.

Equality between two stochastic numbers $X_{1}$ and $X_{2}$ is defined as follows: $X_{1}$ is stochastically equal to $X_{2}$, denoted by $X_{1 \mathrm{~s}}=X_{2}$, if and only if

$$
X_{1 \mathrm{~s}}-X_{2}=\underline{0} \Rightarrow\left|m_{1}-m_{2}\right| \leq \lambda_{\eta} \sqrt{\sigma_{1}^{2}+\sigma_{2}^{2}} .
$$

Definition 5. Order relations between stochastic numbers.

- $X_{1}$ is stochastically greater than $X_{2}$, denoted $X_{1 \mathrm{~s}}>X_{2}$, if and only if

$$
m_{1}-m_{2}>\lambda_{\eta} \sqrt{\sigma_{1}^{2}+\sigma_{2}^{2}}
$$

- $X_{1}$ is stochastically greater than or equal to $X_{2}$, denoted $X_{1 \mathrm{~s}} \geq X_{2}$, if and only if

$$
m_{1} \geq m_{2} \text { or }\left|m_{1}-m_{2}\right| \leq \lambda_{\eta} \sqrt{\sigma_{1}^{2}+\sigma_{2}^{2}}
$$

Based on these definitions the following properties of stochastic arithmetic have been proved [21, 22]:

1. $m_{1}=m_{2} \Rightarrow X_{1 \mathrm{~s}}=X_{2}$.

2. $\mathrm{s}=$ is a reflexive and symmetric relation, but it is not a transitive relation.

3. $X_{1 \mathrm{~s}}>X_{2} \Rightarrow m_{1}>m_{2}$.

4. $m_{1} \geq m_{2} \Rightarrow X_{1 \mathrm{~s}} \geq X_{2}$.

5. $<$ is the opposite of ${ }_{\mathrm{s}}>$.

6. $s>$ is a transitive relation. 
7. $s \geq$ is a reflexive and symmetric relation, but is not a transitive relation.

8. $\underline{0}$ is absorbent; i.e., $\forall X \in \mathbb{S}, \underline{0}_{\mathrm{s}} \times X_{\mathrm{s}}=\underline{0}$.

\subsubsection{Some Algebraic Structures of Stochastic Arithmetic}

As seen above stochastic numbers are Gaussian random variables with a known mean value and a known standard deviation.

It can be seen that algebraic structures close to those existing in the set of real numbers can be developed for stochastic numbers. But this is beyond the scope of this handbook.

As an example a numerical example of the algebraic solution of linear systems of equations with right-hand sides involving stochastic numbers is presented. The aim of this example is to show how a theory can be developed for a better understanding of the properties of the CESTAC method. In particular, it must be noticed that the signs of errors are unknown but when computing with these errors, operations are done with their signs. Consequently, as errors are represented in the theory by standard deviations of Gaussian variables, a sign must be introduced for them. This is done in the same way that intervals are extended to generalized intervals in which the bounds are not ordered. A stochastic number $(m, \sigma)$ for which $\sigma$ may be negative is called a generalized stochastic number.

For a detailed presentation of the theory, see [23-26].

The solution of a linear system with stochastic right-hand sides [24]. We shall use here the special symbol $\dot{+}$ for the arithmetic addition over standard deviations and the special symbol $*$ for the multiplication of standard deviations by scalars. These operations are different from the corresponding ones for numbers but the use of the same symbol $*$ for the multiplication of standard deviations or stochastic numbers by a scalar causes no confusion. The operations + and $*$ induce a special arithmetic on the set $\mathbb{R}^{+}$. We consider a linear system $A x=b$, such that $A$ is a real $n \times n$ matrix and the right-hand side $b$ is a vector of stochastic numbers. Then the solution $x$ also consists of stochastic numbers, and in consequence, all arithmetic operations (additions and multiplications by scalars) in the expression $A x$ involve stochastic numbers; therefore, we shall write $A * x$ instead of $A x$.

Problem.. Assume that $A=\left(a_{i j}\right), i, j=1, \ldots, n, a_{i j} \in \mathbb{R}$ is a real $n \times n$ matrix, and $B=(b, \tau)$ is a $n$-tuple of (generalized) stochastic numbers, such that $b, \tau \in \mathbb{R}^{n}, b=\left(b_{1}, \ldots, b_{n}\right)$, and $\tau=\left(\tau_{1}, \ldots, \tau_{n}\right)$. We look for a (generalized) stochastic vector $X=(x, \sigma), x, \sigma \in \mathbb{R}^{n}$, i.e., an $n$-tuple of stochastic numbers, such that $A * X=B$.

Solution.. The $i$ th equation of the system $A * X=B$ reads $a_{i 1} * x_{1} \dot{+} \cdots+a_{i n} * x_{n}=b_{i}$. Obviously, $A * X=B$ reduces to a linear system $A x=b$ for the vector $x=\left(x_{1}, \ldots, x_{n}\right)$ of mean values and a system $A * \sigma=\tau$ for the standard deviations $\sigma=\left(\sigma_{1}, \ldots, \sigma_{n}\right)$. If $A=\left(a_{i j}\right)$ is non-singular, then $x=A^{-1} b$. We shall next concentrate on the solution of the system $A * \sigma=\tau$ for the standard deviations.

The $i$ th equation of the system $A * \sigma=\tau$ reads $a_{i 1} * \sigma_{1}+\cdots+a_{i n} * \sigma_{n}=\tau_{i}$. It has been proved [21] that this is equivalent to

$$
a_{i 1}^{2} \operatorname{sign}\left(\sigma_{1}\right) \sigma_{1}^{2} \dot{+} \cdots+a_{i n}^{2} \operatorname{sign}\left(\sigma_{n}\right) \sigma_{n}^{2}=\operatorname{sign}\left(\tau_{i}\right) \tau_{i}^{2}, \quad i=1, \ldots, n,
$$

with $\operatorname{sign}\left(\sigma_{j}\right)=1$ if $\sigma_{j} \geq 0$ and $\operatorname{sign}\left(\sigma_{j}\right)=-1$ if $\sigma_{j}<0$.

Setting $y_{i}=\operatorname{sign}\left(\sigma_{i}\right) \sigma_{i}^{2}$ and $c_{i}=\operatorname{sign}\left(\tau_{i}\right) \tau_{i}^{2}$, we obtain a linear $n \times n$ system $D y=c$ for $y=\left(y_{i}\right)$, where $D=\left(a_{i j}^{2}\right)$ and $c=\left(c_{i}\right)$. If $D$ is non-singular, we can solve the system $D y=c$ for the vector $y$ and then obtain the standard deviation vector $\sigma$ by means of $\sigma_{i}=\operatorname{sign}\left(y_{i}\right) \sqrt{\left|y_{i}\right|}$. Thus for the solution of the original problem it is necessary and sufficient that both matrices $A=\left(a_{i j}\right)$ and $D=\left(a_{i j}^{2}\right)$ are non-singular.

Summarizing, to solve $A * X=B$ the following steps are performed:

1. Check the matrices $A=\left(a_{i j}\right)$ and $D=\left(a_{i j}^{2}\right)$ for non-singularity.

2. Find the solution for mean values; i.e., solve the linear system $A x=b$. 
3. Find the solution $y=D^{-1} c$ of the linear system $D y=c$, where $c=\left(c_{i}\right)$ and $c_{i}=\operatorname{sign}\left(\tau_{i}\right) \tau_{i}^{2}$. Compute $\sigma=\operatorname{sign}\left(y_{i}\right) \sqrt{\left|y_{i}\right|}$.

4. The solution of $A * X=B$ is $X=(x, \sigma)$.

\section{Numerical Experiments}

Numerical experiments, using imprecise stochastic data, have been performed to compare the theoretical results with numerical results obtained using the CESTAC method, implemented in the CADNA library (see Section 2.8). As an example, the two solutions obtained for a linear system are reported below.

Let $A=\left\{a_{i j}\right\}$ be a real matrix such that $a_{i j}=i$, if $i=j$ else $a_{i j}=10^{-|i-j|}, i, j=1, \ldots, n$.

Assume that $B$ is a stochastic vector such that the component $B_{i}$ is a stochastic number with a mean value $b_{i}=\sum_{j=1}^{n} a_{i j}$ and a standard deviation for each component equal to $1 \cdot e-4$. The centers $x_{i}$ of the components of the solution are thus close to 1 and present no difficulty for their computation.

The theoretical standard deviations are obtained according to the method described in the previous section. First, matrix $D$ is computed from the matrix $A$, then $D y=c$ is solved, and then $\sigma_{i}=\operatorname{sign}\left(y_{i}\right) \sqrt{\left|y_{i}\right|}$ is computed.

For a correct comparison of the solution provided by the CADNA library and the theoretical solution, accurate values for the standard deviations are obtained as follows. Twenty different vectors $b^{(k)}, k=$ $1, \ldots, 20$, for the right-hand side have been randomly generated, and the corresponding twenty systems $A * X=B^{(k)}$ have been solved. For each component of $B^{(k)}$, the standard deviation of the $N=3$ samples has been computed with the CADNA software and then the mean value of the standard deviations has been computed for each component and presented in Table 2.1 .

As we can see in Table 2.1, the theoretical standard deviations and the computed values are very close.

To conclude this subsection we comment that the theoretical study of the properties of stochastic numbers allows us to obtain a rigorous abstract definition of stochastic numbers with respect to the operations of addition and multiplication by scalars. This theory also allows the solution of algebraic problems with stochastic numbers. Moreover, this provides a possibility of comparing algebraically obtained results with practical applications of stochastic numbers, such as those provided by the CESTAC method [27].

Remark. The authors are grateful to Professor S. Markov from the Bulgarian Academy of Sciences and to Professor J.L. Lamotte from University Pierre et Marie Curie (Paris, France) for their contribution to the above section.

Table 2.1 Theoretical and computed standard deviations

\begin{tabular}{ccc} 
Component & $\begin{array}{c}\text { Theoretical standard } \\
\text { deviations, } x^{\prime \prime}\end{array}$ & $\begin{array}{c}\text { Computed standard } \\
\text { deviations }\end{array}$ \\
\hline 1 & $9.98 \mathrm{e}-05$ & $10.4 \mathrm{e}-05$ \\
2 & $4.97 \mathrm{e}-05$ & $4.06 \mathrm{e}-05$ \\
3 & $3.32 \mathrm{e}-05$ & $3.21 \mathrm{e}-05$ \\
4 & $2.49 \mathrm{e}-05$ & $2.02 \mathrm{e}-05$ \\
5 & $1.99 \mathrm{e}-05$ & $1.81 \mathrm{e}-05$ \\
6 & $1.66 \mathrm{e}-05$ & $1.50 \mathrm{e}-05$ \\
7 & $1.42 \mathrm{e}-05$ & $1.54 \mathrm{e}-05$ \\
8 & $1.24 \mathrm{e}-05$ & $1.02 \mathrm{e}-05$ \\
9 & $1.11 \mathrm{e}-05$ & $0.778 \mathrm{e}-05$ \\
10 & $0.999 \mathrm{e}-05$ & $0.806 \mathrm{e}-05$ \\
\hline
\end{tabular}




\subsection{Validation and Implementation of the CESTAC Method \\ 2.5.1 Validation and Reliability of the CESTAC Method}

The theoretical validation of the CESTAC method is therefore established if and only if the two previous hypotheses hold. But its efficiency in scientific codes can be guaranteed only if its underlying hypotheses hold in practice:

- Concerning hypothesis 1 , because of the use of the random rounding mode, round-off errors $\alpha_{i}$ are random variables; however, in practice they are not rigorously centered and in this case Student's test leads to a biased estimation of the computed result. It may be thought that the presence of a bias seriously jeopardizes the reliability of the CESTAC method. In fact it has been proved in [13] that it is the ratio $q$ of the bias divided by the standard deviation $\sigma$ which is the key of the reliability of equation (12).

It is shown in $[13,21]$ that a magnitude of $q$ of several tens only induces an error less than one decimal significant digit on $C_{\bar{R}}$ computed with equation (12). This great robustness of equation (12) is due first to the use of the logarithm and second to the natural robustness of Student's test. Consequently in practice even if hypothesis 1 is not exactly satisfied, it is not a drawback for the reliability of equation (12).

- Concerning hypothesis 2, the approximation to first order only concerns multiplications and divisions, because in formulas (5) and (6) for round-off errors in additions or subtractions, the second-order terms, i.e., those in $2^{-2 p}$, do not exist. For the first-order approximation to be legitimate, it is shown in $[2,20]$ that if $\varepsilon_{1}$ and $\varepsilon_{2}$ are, respectively, the absolute round-off errors on the operands $X_{1} \in \mathbb{F}$ and $X_{2} \in \mathbb{F}$, the following condition must be verified:

$$
\operatorname{Max}\left[\left|\frac{\varepsilon_{1}}{X_{1}}\right|,\left|\frac{\varepsilon_{2}}{X_{2}}\right|\right] \ll 1 .
$$

Hence, the more accurate the computed results, the more legitimate the first-order approximation. However, if a computed result becomes non-significant, i.e., if its round-off error is of the same order of magnitude as itself, then the first-order approximation may not be legitimate.

In other words, with the use of the CESTAC method hypothesis 2 holds when

1. The operands of any multiplication are both significant.

2. The divisor of any division is significant.

As a consequence, validation of the CESTAC method requires during the run of the code to control steps (1) and (2). Indeed if (1) or (2) is not satisfied, this means that hypothesis 2 has been violated and then the results obtained with equation (12) must be considered as unreliable. This control is achieved with the concept of the computational zero described in Section 2.6. This control is in practice performed by the CADNA library, which is sketched in Section 2.8 .

\subsubsection{Implementation of the CESTAC Method}

The two main features of the CESTAC method are as follows:

- The random rounding for each arithmetical operation, which consists in randomly choosing either the result rounded to up $\rho^{+}$or the result rounded to down $\rho^{-}$.

- Performing the $N$ runs of code.

To set these features in context we must consider the period pre-1988 when FP arithmetic was machine dependent and post-1988 when it was standardized by IEEE. 


\section{Asynchronous Implementation}

Before 1988, FP arithmetic was highly computer dependent. Scientific computers as IBM, CDC, and CRAY worked with different rounding modes either with a chopping mode (rounding to zero) or with a rounding to the nearest mode. Sometimes, even on the same computer some arithmetic operations were performed with the chopping mode and others with the rounding to the nearest mode.

At this time an implementation which violates the hypotheses of the method has been used in a software named Prosolver [28]. As a consequence this flawed software has been the origin of some criticisms as in $[29,30]$, which have been erroneously attributed to the method. This implementation has also been used later in the Monte Carlo arithmetic (see [31-33]).

In this implementation, which is called 'asynchronous implementation,' the $N$ runs of a code were performed independently. This means that the code was first run to completion and then it was run a second time and so on until the $N$ th run. In addition, in the software Prosolver, the random rounding mode consisted in randomly adding \pm 1 or 0 to the last bit of every FP operation result. This random rounding mode is unsatisfactory because, even when the result of an FP operation is an exact FP value, it is increased or decreased by one unit on the last position (ulp). The main criticisms of this implementation were that the random rounding used as defined before violates theorems about exact rounding, and when a computation is virulently unstable but in a way that almost always diverges to the same wrong destination, such a randomized recomputation almost always yields the same wrong result.

The correct implementation is now described in the following section.

\section{Correct Synchronous Implementation}

It is only since 1990 that the standard IEEE 754 FP arithmetic has been available to users. Around the same time scientific languages began to provide users with the capability of overloading operators. With IEEE 754 arithmetic and the overloading statements it is easy to implement the CESTAC method correctly.

- A correct random rounding mode

It was proposed in Section 2.3.2 to choose $\rho^{-}$or $\rho^{+}$as result of FP operator. In practice we use the IEEE 754 rounding toward $+\infty$ and toward $-\infty$. Rounding occur only when an arithmetic operation has a result that is not exact. Therefore no artificial round-off error is introduced in the computation. The choice of the rounding is at random with an equal probability for the $(N-1)$ first samples, with the last one chosen as the opposite of the $(N-1)$ th sample.

With this random rounding the theorems on exact rounding are respected.

- Synchronous runs

We have seen previously that to control the reliability of the CESTAC method it is absolutely necessary to detect during the run of the code the emergence of computational zeroes. To achieve this it suffices to use the synchronous implementation which consists of performing each FP operator $N$ times with the random rounding mode before performing the next operation. Thus everything proceeds as if $N$ identical codes were running simultaneously on $N$ synchronized computers each using the random rounding mode. Thus for each numerical result we have $N$ samples, from which with equation (12) the number of significant decimal digits of the mean value, considered as the computed result, is estimated. With this implementation a DSA may be defined, allowing during the run of the code to control dynamically the reliability of the CESTAC method.

Thus it is possible dynamically to

- control the round-off error propagation of each FP operation,

- detect a loss of accuracy during the computation,

- control the branching statements, and

- detect a violation of hypothesis 2 which guarantees the reliability of the method.

\subsection{Discrete Stochastic Arithmetic (DSA)}

The concept of the computational zero and the synchronous implementation of the CESTAC method leads to operations on $\mathrm{N}$-tuples as referred to discrete stochastic numbers. Operation on these numbers is 
also termed DSA. The salient properties of this arithmetic, which is detailed in $[16,17,34]$, are presented here.

From the granular computing point of view, a discrete stochastic number is a granule and the DSA is a tool for computing granules.

\subsubsection{Discrete Stochastic Arithmetic Operators}

Definition 6. Discrete stochastic numbers (granules).

A discrete stochastic number is an $N$-tuple formed by the $N$ samples provided by the synchronous implementation of the CESTAC method.

Definition 7. Discrete stochastic arithmetic (tools working on granules).

DSA operates on discrete stochastic numbers. The result of the four discrete stochastic operators is by definition the result of the corresponding arithmetic operation provided by the CESTAC method. Let $X, Y$, and $Z$ be discrete stochastic numbers, and let $\diamond$ be an FP arithmetic operator $\diamond \in[\oplus, \ominus, \otimes, \oslash]$, as defined in Section 2.2,

$$
X=\left(X_{1}, \ldots, X_{N}\right), \quad Y=\left(Y_{1}, \ldots, Y_{n}\right), \quad Z=\left(Z_{1}, \ldots, Z_{N}\right) .
$$

Then any of the four stochastic arithmetic operations ${ }_{\mathrm{s}}+,{ }_{\mathrm{s}}-,{ }_{\mathrm{s}} \times,{ }_{\mathrm{s}} /$, denoted ${ }_{s} \diamond$, is defined as

$$
Z=X_{s} \diamond Y \Rightarrow Z=\left(\left(X_{1} \diamond Y_{1}\right)^{ \pm}, \ldots,\left(X_{N} \diamond Y_{N}\right)^{ \pm}\right)
$$

where \pm means that the FP operation $\diamond$ has been randomly performed with the rounding toward $+\infty$ or toward $-\infty$, as explained previously. Thus any discrete stochastic operator provides a result that is an $\mathrm{N}$-tuple obtained from the corresponding FP operator operating on the components of the two operands the result of which is rounded at random toward $+\infty$ or $-\infty$.

Remark. To simplify the notations the ones for the discrete stochastic operators are chosen to be the same as those for the (continuous) stochastic operators.

Then with DSA it is straightforward using equation (12) to estimate the number of significant decimal digit of any result produced by a DSA operator.

Definition 8. Discrete stochastic zero (computational zero) [15].

Any discrete stochastic number $X=\left(X_{1}, X_{2}, \ldots, X_{N}\right)$ is a discrete stochastic zero, also called computational zero, denoted @.0, if one of the two following condition holds:

1. $\forall i, X_{i}=0, \quad i=1, \ldots, N$.

2. $C_{\bar{X}} \leq 0$, where $C_{\bar{X}}$ is obtained from equation (12).

\subsubsection{Discrete Stochastic Relations (Tools Working on Granules)}

From the concept of the discrete stochastic zero @.0, discrete stochastic relations can now be defined.

Let $X, Y$ be discrete stochastic numbers, it is possible to define equality and order relations for these numbers. They are called discrete stochastic equality and discrete order relations and are defined as follows. 
Definition 9. Discrete stochastic equality denoted by $\mathrm{s}_{\mathrm{s}}=$.

The discrete stochastic equality is defined by

$$
X_{\mathrm{s}}=Y \quad \text { if } \quad Z=X_{\mathrm{s}}-Y=@ .0 .
$$

Definition 10. Discrete stochastic inequalities denoted by ${ }_{\mathrm{s}}>$ and $_{\mathrm{s}} \geq$.

These are defined by

$$
\begin{array}{llll}
X_{\mathrm{s}}>Y & \text { if } \quad \bar{X}>\bar{Y} \quad \text { and } & X_{\mathrm{s}}-Y \neq @ .0 ; \\
X_{\mathrm{s}} \geq Y & \text { if } \quad \bar{X} \geq \bar{Y} \quad \text { or } & X_{\mathrm{s}}-Y=@ .0 .
\end{array}
$$

With this DSA it is possible during the execution of a code to follow the round-off error propagation, detect numerical instabilities, check branchings, and check hypotheses that guarantee the reliability of equation (12).

\subsection{Taking into Account Data Errors}

In real-life problems, data often come from measurements and thus contain errors issuing from sensors. Most of the time data errors may be considered as centered Gaussian random variables. It is then absolutely necessary to estimate the effect of these errors on the numerical results provided by DSA.

In a similar fashion to estimating equation (11), let us consider a finite sequence of $v$ arithmetic operations, providing a single result $r$ and requiring $n d$ uncertain data $d_{i}, i=1, \ldots, n d$. Let $\delta_{i}$ be the data error on each $d_{i}$. These $\delta_{i}$ 's may be considered as Gaussian variables with a standard deviation of $\sigma_{i}$. It has been proved $[3,35]$ that when the previous finite sequence is performed with DSA, each data $D_{i}, i=1, \ldots, n d$, is defined by

$$
D_{i}=d_{i}\left(1+2 \theta \sigma_{i}\right)
$$

$\theta$ being a random number uniformly distributed on ] $-1,+1$ [, then each $N$-tuple of the computer result $R$ may be modeled by a Gaussian random centered variable

$$
R \simeq r+\sum_{i=1}^{n d} v_{i}(d) 2^{-p} \delta_{i}+\sum_{i=1}^{v} g_{i}(d) 2^{-p} z_{i}
$$

$v_{i}(d)$ being quantities depending exclusively on the data and on the code. This formula is an extension of equation (11). Indeed the first quantity represents the error coming from uncertainties of data and the second represents the round-off error propagation. Then to estimate the number of significant decimal digits in the computed result $\bar{R}$ it suffices to use equation (21). In this estimation both errors (uncertainties of data and round-off error) have been taken in account.

In the framework of granular computing each data item $D_{i}$ is a granule elaborated from (20), which is an operand for the DSA operators.

\subsection{The CADNA Library [36]}

The CADNA library has been written in Fortran, $\mathrm{C}++$, and ADA. It is presented in detail in [20]. It is the Fortran version that is described here. The CADNA library automatically implements DSA in any Fortran code. For CADNA Fortran and CADNA ADA, $N=3$ has been chosen. But for CADNA $\mathrm{C}++$, the value of $N$ must be chosen by the user. Furthermore, the probability is here chosen to the classical level of $\eta=0.95$. As seen in the beginning, in equation (12) for $N=3$ and $1-\eta=0.05$ 
the value of Student's table for $N-1=2$ degrees of freedom is $\tau_{\eta}=4.303$. Thus the CADNA library enables a user to run a scientific code with DSA on a conventional computer without having to rewrite or even substantially modify the Fortran source code. A new 'stochastic number' type has been created, which is a triplet (because $N=3$ ), each component being a sample provided by the random rounding.

All the Fortran arithmetic operators, $+,-, *, /$, have been overloaded, so that when such an operator is performed, the operands and the result are stochastic numbers. In the same way the relational operators such as $==,>, \geq,<, \leq$ have also been overloaded, satisfying the properties of the discrete stochastic relations.

Moreover, all the standard functions defined in Fortran 77 have also been overloaded. Similarly, the printing statement has been modified and gives the computer result written only with its exact number of significant digits, estimated by equation (12). Furthermore, in order to estimate the effect of data errors on a result provided by the computer, a special function has been created that allows the user to introduce uncertainties into these data. This function must always be used associated with assignment statements when data are not exact FP values.

The modifications that the user has to make to the Fortran source are mainly to change the declaration statements of real type to stochastic type, and the input-output statements. Thus, see [36], when a modified Fortran source combined with the CADNA library is run, it is as if $(N=3)$ identical codes were simultaneously run on $N$ synchronized computers, each of them using the random rounding mode. So round-off error propagation can be analyzed step by step and then any numerical anomaly can be detected.

One major feature of the CADNA library is that this dynamical analysis is performed during the execution of code. As soon as a numerical anomaly is detected, a warning is written to a special CADNA file.

These warnings are divided into two categories: those concerning the reliability of the results provided and those concerning the numerical debugging of the code.

- Concerning the reliability of the results, the warnings are

- unstable multiplication (the operands of the multiplication are computational zeroes),

- unstable power (the operand of the power is a computational zero), and

- unstable division (the divisor is a computational zero).

- Concerning the debugging of the code, the warnings are

- instabilities in functions ( $S I G N, M O D, D I M, L O G, S I N, \ldots$ ),

- computational zero detected in a branching, and

- sudden loss of accuracy.

When a code has been instrumented with the CADNA library and run, the user must always consult the special CADNA file. If it is empty, this means that no anomaly has been detected and that the computed results provided by the code are reliable and that the number of significant decimal digits of each of them is correctly estimated up to 1 .

If the special CADNA file contains warnings, the following two cases must be considered:

1. One or several warnings belonging to the first category appear in the file. This means that hypothesis 2 has been violated and so the results provided by the code must be considered as unreliable.

2. One or several warnings belonging to the second category appear. This means that instabilities have been detected. In this case, the user is able, with the use of the debugger, to identify the statement in which the anomaly has appeared. The user must then try to improve the stability of the code, for instance, by replacing unstable formulas by more stable ones.

With the special CADNA file the user knows the numerical behavior of the code and then may conclude about the reliability of results obtained. 


\subsection{The Use of the CADNA Library}

In scientific computing, numerical methods are used for solving problems on a computer. These numerical methods can be classified in three categories: finite methods, iterative methods, and approximate methods.

\subsubsection{Finite Methods}

A method of this class consists of a finite ordered sequence of arithmetic operations and branchings depending on some criteria, e.g., elimination methods for linear systems and more general scientific computations that involve a succession of algebraic formulas. As shown earlier, when these methods are performed on a computer with the usual FP arithmetic, false results may be obtained without any warning. But when these methods are performed with DSA and using the CADNA library, numerical instabilities are detected and the result is provided with its accuracy. To illustrate this, consider the following example.

Example 1. This example has been proposed in [29, 30]. It is an adaptation from another example [37] and consists in computing the result of the following formula;

$$
t=L-\frac{(M-N /(L-(M-N / z) /(L-(M-N / y) / z)))}{(L-(M-N /(L-(M-N / y) / z)) /(L-(M-N / Z) /(L-(M-N / y) / z)))}
$$

with

$$
\begin{array}{lll}
L=a+b+c & M=a(b+c)+b c & N=a b c \\
x=\frac{b+c}{2} & y=\frac{b \cdot b+c \cdot c}{b+c} & z=L-\frac{M-N / x}{y} \\
a=3.10^{8} & b=6 & c=5
\end{array}
$$

The exact result is $t=\left(b^{7}+c^{7}\right) /\left(b^{6}+c^{6}\right)$; i.e., $t=358061 / 62281=5.7491209197$.

With IEEE standard arithmetic and any of its rounding mode, the obtained result is always $t=3.0 \times 10^{8}$ and of course the user is not informed that this result is false.

With the Prosolver software the result obtained is again $t=3.0 \times 10^{8}$. So Prosolver has also failed.

With the CADNA library the result is the same, $t=3.0 \times 10^{8}$, but the special CADNA file contains six 'unstable division' warnings. As explained previously, this means that hypothesis 2 has been violated. Thus the provided result is not reliable and must be considered as an incorrect result. CADNA has not failed.

Example 2. This system of linear equations has been proposed by J.H. Wilkinson and can be found in [14]. It is defined as follows: $W_{n} \cdot \mathcal{X}=\mathcal{B}$,

with $W_{n}=\left(w_{i, j}\right), \quad i, j=1, \ldots, n$, with

$$
\left\{\begin{array}{l}
w_{i, i}=1.0 \\
w_{i, j}=-1.0 \text { for } i>j \\
w_{i, j}=0.0 \text { for } i<j \\
w_{i, n}=1.0 \text { for } i \in[1, n-1] \\
w_{n, n}=\alpha=0.9
\end{array}\right.
$$

In this system the diagonal and the nth column and are $(1,1, \ldots \alpha)$, the elements of the upper triangular sub-matrix are null except the last column, and those of the lower triangular sub-matrix are -1 . The $n$ elements of right-hand side $\mathcal{B}$ are equal to 1 . 
It is easy to show that the exact solution of this system, which is not ill conditioned, is

$$
\left\{\begin{array}{l}
x_{i}^{*}=-2^{i-1} \frac{1-\alpha}{\Delta^{*}} \quad i=[1, n-1] \\
x_{n}^{*}=\frac{2^{n-1}}{\Delta^{*}}
\end{array}\right.
$$

$\Delta^{*}=2^{n-1}-1+\alpha, \Delta^{*}$ being the determinant of the matrix.

This system has been solved using Gaussian elimination method with partial pivoting, first with the IEEE 754 standard double precision and them with the CADNA library, for $n \in[30,35,40,45,50]$.

The determinant computed with both the IEEE 754 standard and the CADNA library yields results correct to 15 significant decimal digits.

Concerning the solution $X_{i}, i=1, n-1$, we find the following results which are detailed in Table 2.2.

- With the IEEE 754 standard some of the last digits are false, but of course the user is not informed of the failure.

- With the CADNA library, only the $\mathcal{N}$ decimal digits estimated to be exact up to 1 by the software are provided. It can be seen in Table 2.2 that these are in perfect agreement with the number of exact digits, $\mathcal{N}^{*}$, obtained by comparing the CADNA solution to the exact solution $x_{i}^{*}, i=1, n-1$.

The following example concerns a problem with uncertain data solved by the CADNA library. To perturb the data, CADNA uses a special function constructed according to formula (20).

Example 3. Study of the sensitivity of a determinant to the coefficients of the matrix. Let us consider the determinant proposed in [38]:

$$
\Delta=\left|\begin{array}{rrr}
-73 & 78 & 24 \\
92 & 66 & 25 \\
-80 & 37 & 10
\end{array}\right| .
$$

The exact value of this determinant is $\Delta=1$.

When this determinant is computed with IEEE 754 FP arithmetic in double precision using different rounding modes, the results obtained are as follows:

- with the rounding to nearest mode $\Delta=0.9999999999468869$,

- with the rounding to zero mode $\Delta=0.9999999999468865$,

- with the rounding to $-\infty$ mode $\Delta=0.9999999999 \underline{894979}$, and

- with the rounding to $+\infty$ mode $\Delta=1.000000000 \underline{747207}$.

The underlined digits are false but obviously the user is not aware of this fact. When the determinant is computed with the CADNA library, the result is $\Delta=1.000000000$. Note that the result is printed with only ten digits, which is the best accuracy which can be obtained.

Table 2.2 Accuracy of the solution of system (23) for different size $n$

\begin{tabular}{cccc}
\hline$n$ & $\begin{array}{c}\text { Number of false } \\
\text { last decimal digits } \\
\text { (IEEE 754 standard) }\end{array}$ & $\begin{array}{c}\text { Number of } \\
\text { decimal digits, } \mathcal{N} \\
\text { (CADNA library) }\end{array}$ & $\begin{array}{c}\text { Number of exact } \\
\text { decimal digits, } \mathcal{N}^{*}\end{array}$ \\
\hline 30 & 9 & 6 & 6 \\
35 & 10 & 4 & 5 \\
40 & 12 & 3 & 3 \\
45 & 13 & 1 & 2 \\
50 & 15 & 0 & 0 \\
\hline
\end{tabular}


Table 2.3 Number of exact decimal digits of $\Delta$ as a function of $\varepsilon$

\begin{tabular}{ccccccc}
\hline$\varepsilon$ & $10^{-15}$ & $10^{-13}$ & $10^{-11}$ & $10^{-9}$ & $10^{-7}$ & $10^{-5}$ \\
$\mathcal{N}$ & 10 & 8 & 6 & 4 & 2 & 0 \\
\hline
\end{tabular}

Suppose now that coefficients $a_{12}=78$ and $a_{33}=10$ of the matrix are uncertain data. This means that they both contain a relative error $\varepsilon$, which is taken here to be the same. In other words,

$$
a_{12} \in[78-78 \varepsilon, 78+78 \varepsilon] \text { and } a_{33} \in[10-10 \varepsilon, 10+10 \varepsilon] .
$$

The CADNA library, as explained above, is an effective tool for estimating the influence of data uncertainties on the computed determinant.

Table 2.3 presents the number of exact decimal digits, $\mathcal{N}$, provided by CADNA in the computed determinant (25) as a function of $\varepsilon$, which determines the uncertainty of $a_{12}$ and $a_{33}$.

From these results it clearly appears that if the magnitude of uncertainty in the coefficients is greater than or equal to $10^{-5}$, then the determinant cannot be computed since the result obtained is not significant.

\subsubsection{Iterative Methods}

From the mathematical standpoint, these methods, starting from an initial point $x_{0}$ considered as an approximation of the solution to the problem to be solved, consist in computing a sequence $x_{1}, x_{2}, \ldots, x_{k}$ that is supposed to converge to the solution.

So, let us consider here an iterative sequence defined by

$$
x_{k+1}=\varphi\left(x_{k}\right) \quad \mathbb{R}^{m} \stackrel{\varphi}{\longrightarrow} \mathbb{R}^{m} .
$$

If the method is convergent, then $\exists x: x=\lim _{k \rightarrow \infty} x_{k}$.

From the computational point of view, this limit cannot be reached, and consequently a termination criterion is used to stop the iterative process, such as

$$
\text { if }\left\|X_{k}-X_{k-1}\right\| \leq \varepsilon\left\|X_{k}\right\| \text { then stop } \quad X_{k} \in \mathbb{F}^{m},
$$

where $\varepsilon$ is an arbitrary positive value. It is clear that this termination criterion is not satisfactory for two reasons.

If $\varepsilon$ is too large then the sequence is broken off before a good approximation to the solution is reached. On the contrary if $\varepsilon$ is too small, then many useless iterations are performed, without improving the accuracy of the solution because of round-off error propagation. Moreover each $X_{k}$ has only a certain number of significant decimal digits. If the $\varepsilon$ selected is less than the accuracy of $X_{k}$, this termination criterion is no longer meaningful. Two problems then arise.

1. How can the iterative process be stopped correctly?

2. What is the accuracy of the computed solution provided by the computer?

With the use of the CADNA library, thanks to the properties of DSA, it is possible to define new termination criteria, depending on the problem to be solved, which stop the iterative process as soon as a satisfactory computational solution is reached.

Indeed two categories of problems exist:

1. those for which there exists some function which is null at the solution of the problem. The solution of a linear or non-linear system or the search of an optimum for a constrained or non-constrained problem belong to this category;

2. those for which such a function does not exist. Such is the computation of the sum of a series. 
For the first category the termination criterion is called 'optimal termination criterion.' It acts directly on functions which must be null at the solution of the problem. For example, from the mathematical standpoint if $x_{s} \in \mathbb{R}^{m}$ is the solution of a linear system then $A \cdot x_{s}-B=0$. The optimal termination criterion consists in stopping the iterative process on the $k$ th iterations if and only if $A * X_{k}-B=@ .0$ (@.0 being the computational zero).

For the second category the usual termination criterion is replaced by

$$
\text { if }\left\|X_{k \mathrm{~s}}-X_{k-1}\right\|=@ \text {. } 0 \quad \text { then stop. }
$$

With this termination criterion the arbitrary value $\varepsilon$ is eliminated.

\section{Example 4. Jacobi's iterations.}

To illustrate the fact that the choice of $\varepsilon$ in the classical termination criterion may be difficult and the efficiency of the optimal termination criterion, let us consider the following linear system $A X=B$ of dimension 25 with

$$
\begin{aligned}
& a_{i j}=\frac{1}{i+j-1} \text { for } i, j=1, \ldots, n \text { and } i \neq j \\
& a_{i i}=1 .+\sum_{j=1}^{i-1} a_{i j}+\sum_{j=i+1}^{n} a_{i j} \quad \text { for } i=1, \ldots, n \\
& b_{i}=\sum_{j=1}^{n} a_{i j} z_{j} \quad \text { for } i=1, \ldots, n, \quad \text { with } \quad z_{j}=3^{j-1} \times 2^{-10}, \quad j=1, \ldots, n .
\end{aligned}
$$

As the diagonal of $A$ is dominant Jacobi's iterations are always convergent.

The exact solution is $x_{j}=3^{j-1} \times 2^{-10}$.

System (26) was first solved using standard IEEE 754 double-precision floating-point arithmetic (DPFP) with several values of $\varepsilon$ and then with the CADNA double-precision DSA. The results are the following:

- With DPFP and the classical termination criterion with $\varepsilon=10^{-4}$, the last unknown $x_{25}$ has been computed with the maximum accuracy (15 decimal digits) and the accuracy decreases from component to component until it reaches 3 decimal digits on the first component $x_{1}$.

- With $\varepsilon<10^{-4}$, the test is never satisfied and the iterations stop on a predefined arbitrary maximum number of iterations. Hence a great number of useless iterations are computed, without improving the accuracy of the solution. For example with $\varepsilon=10^{-5}$, the process is stopped after 10,000 iterations and the accuracy is identical to the one obtained with $\varepsilon=10^{-4}$ (see Table 2.4).

- On the contrary with $\varepsilon=10^{-3}$, the process is stopped too soon (419 iterations) and $x_{1}$ and $x_{25}$ are obtained with, respectively, 2 and 14 decimal digits.

- Moreover in all cases with IEEE 754 FP arithmetic the number of significant decimal digits of each unknown cannot be obtained.

- On the contrary with the use of the CADNA library and the optimal termination criterion defined above the process is stopped as soon as a satisfactory solution is obtained, 459 iterations in this case, and the number of exact decimal digits, up to one, of each component is provided.

The number $\mathcal{N}$ of decimal digits thus obtained for system (26) with the initialization

$$
x_{i}^{0}=15
$$

are given in Table 2.4.

Table 2.4 Number of exact decimal digits in the solution of system (26) with the CADNA library

\begin{tabular}{lllllllllllllllllllllllllll}
\hline$i$ & 1 & 2 & 3 & 4 & 5 & 6 & 7 & 8 & 9 & 10 & 11 & 12 & 13 & 14 & 15 & 16 & 17 & 18 & 19 & 20 & 21 & 22 & 23 & 23 & 25 \\
$\mathcal{N}$ & 3 & 4 & 4 & 5 & 6 & 6 & 6 & 7 & 8 & 9 & 9 & 10 & 10 & 11 & 11 & 12 & 12 & 13 & 13 & 14 & 14 & 15 & 15 & 15 & 15 \\
\hline
\end{tabular}


In fact, as shown in [39] the optimal termination criterion which consists in testing the residual and the usual criterion which consists in testing the difference between two iterates are closely connected in the case of Jacobi's method because

$$
X_{k+1}-X_{k}=D^{-1}\left(B-A X_{k}\right)
$$

matrix $D$ being the diagonal of $A$.

This is perfectly verified with the CADNA library. In fact when the termination criterion is the stochastic equality of two successive vector iterates, the process is stopped at the 460th iteration and the accuracy of the solution is also the same as the one reported in Table 2.4.

\subsubsection{Approximate Methods}

From the mathematical standpoint, these methods provide only an approximation of the solution. This category contains, e.g., numerical computation of derivatives, numerical integration, and numerical solution of differential or partial differential equations.

When these methods are run on a computer, they always provide a solution containing an error $e_{\mathrm{g}}$, which is a combination of the method error $e_{\mathrm{m}}$ inherent in the employed method and the error due to the propagation of round-off errors called computation error $e_{\mathrm{c}}$.

It is well known that the method error $e_{\mathrm{m}}$ is an increasing function of the discrete step size $h$. On the contrary the computation error $e_{\mathrm{c}}$ is an increasing function of the inverse $\frac{1}{h}$ of the step size. This means that $e_{\mathrm{m}}$ and $e_{\mathrm{c}}$ act in the opposite way and consequently the global error $e_{\mathrm{g}}$ is a function which has a minimum for some value of $h$. Thus the best approximation of the solution that can be obtained on a computer corresponds to an optimal discrete step size $h^{*}$, such that $\mathrm{d} e_{\mathrm{g}} / \mathrm{d} h=0$. Obviously, it is impossible to establish a general methodology to estimate $h^{*}$, because the method error $e_{\mathrm{m}}$ is specific to the method. Yet most of the time for a specific method, $e_{\mathrm{m}}$ can be estimated. Furthermore, $e_{\mathrm{c}}$ can be estimated using the CADNA library. Then in many cases, it is possible to estimate $h^{*}$ [17].

To illustrate this, let us consider the following example, which is a simple solution of a differential equation using Euler's method.

Example 5. The differential equation is

$$
y^{\prime}=e^{x} y+x y-(x+1) e^{-x}-1 .
$$

With the initial condition $y(0)=1$, the exact solution is $y(x)=e^{-x}$.

The computation of the optimal step size for each interval $\left[x_{k}, x_{k+1}\right]$ requires three phases:

1. the estimation of the round-off error $e_{\mathrm{c}}$,

2. the evaluation of the truncation error (method error) $e_{\mathrm{m}}$, and

3. the computation of the optimal step size.

The estimation of the round-off error $e_{\mathrm{c}}$ is obtained using the CADNA library. Indeed a special function called nb-significant digits(x) of this library returns the number of significant digits in a stochastic argument $x$. This number is an integer $n$ obtained from equation (12) rounded down. The estimation of the round-off error is then computed by equation (28).

$$
e_{\mathrm{c}}=10^{-(n+1)} \text {. }
$$

The estimation of the truncation error $e_{\mathrm{m}}$ at each step for Euler's method is well known and is given by

$$
e_{\mathrm{m}}=2\left|y_{1}-y_{2}\right| \text {. }
$$


Table 2.5 Solution of equation (27) with different step size

\begin{tabular}{lccccc}
\hline$x$ & Exact solution $y^{*}$ & $h=10^{-1}$ & $h=10^{-3}$ & $h=10^{-6}$ & Optimal $h$ \\
\hline 0.0 & 1.0 & 1.0 & 1.0 & 1.0 & 1.0 \\
0.1 & 0.905 & 0.900 & 0.905 & 0.905 & 0.905 \\
0.2 & 0.819 & 0.809 & 0.819 & 0.818 & 0.819 \\
0.3 & 0.741 & 0.726 & 0.741 & 0.740 & 0.741 \\
0.4 & 0.670 & 0.649 & 0.670 & 0.670 & 0.670 \\
0.5 & 0.607 & 0.578 & 0.606 & 0.606 & 0.607 \\
0.6 & 0.549 & 0.511 & 0.548 & 0.550 & 0.548 \\
0.7 & 0.497 & 0.447 & 0.496 & 0.499 & 0.496 \\
0.8 & 0.449 & 0.384 & 0.448 & 0.453 & 0.447 \\
0.9 & 0.407 & 0.320 & 0.405 & 0.413 & 0.404 \\
1.0 & 0.368 & 0.250 & 0.366 & 0.380 & 0.366 \\
\hline
\end{tabular}

$y_{1}$ is the value of $y\left(x_{k}+h_{k}\right)$ integrated over the interval $\left[x_{k}, x_{k}+h_{k}\right]$ with step size $h_{k}$, while $y_{2}$ is the value of $y\left(x_{k}+h_{k}\right)$ integrated over the same interval $\left[x_{k}, x_{k}+h_{k}\right]$ with step size $\frac{1}{2} h_{k}$. Of course, $e_{\mathrm{m}}$ may not be less than $e_{\mathrm{c}}$ because it is also computed with the same computer.

The optimal step size $h^{*}$ can then be obtained with a simple minimization method.

The results obtained in single precision using CADNA are presented in Table 2.5.

From the results of Table 2.5 it clearly appears that if the step size is too large $(h=0.1)$ or too small $\left(h=10^{-6}\right)$, only one or two decimal digits are obtained in the solution, while with the optimal step size the solution is computed with two or three exact digits.

Here, a very simple method to estimate the optimal step size has been presented, but more sophisticated methods have also been developed in [27].

\subsection{Can the CADNA Library Fail?}

To answer to this question, imagine a computation such that only one or two rounding errors are the dominant contribution to the final error. This is the case in example 1 and example 2. Concerning example 1 which has been specially created to jeopardize the stochastic approach of FP computation, it can be shown experimentally that as the number of samples $N$ increases the percentage of failure decreases. This percentage which is presented in Table 2.6 is in total agreement with the approximation of the mean value and standard deviation of an unknown Gaussian distribution by those of empirical values which is used in equation (12).

Concerning example 2, during the Gaussian elimination there is no round-off error propagation except for the last pivot $a_{n, n}$, because all the other results are integer values which are exact FP values.

It is exactly the same thing for the computation of the $n$ elements of the right-hand side $\mathcal{B}$ which are also exact FP values.

The values of the last pivot $a_{n, n}$ and $b_{n}$ are

$$
a_{n, n}=\alpha+2^{n-1}-1 \text { and } b_{n}=2^{n-1} .
$$

Table 2.6 Percentage of failure as a function of the number $N$ of samples in example 1

\begin{tabular}{cccccc}
\hline$N$ & 3 & 4 & 5 & 7 & 10 \\
$\%$ & 10 & 5 & 3 & 1 & 0 \\
\hline
\end{tabular}


Table 2.7 Percentage of failures as a function of $n$ and $N$ in example 2

\begin{tabular}{rccccc}
\hline$n$ & $N=3$ & $N=4$ & $N=5$ & $N=6$ & $N=7$ \\
\hline 5 & 5 & 3 & 2 & 0 & 0 \\
25 & 10 & 5 & 3 & 1 & 0 \\
45 & 10 & 6 & 4 & 3 & 0 \\
\hline
\end{tabular}

The larger the $n$, the closer $x_{n}$ is to 1 .

Furthermore, $x_{n}=b_{n} / a_{n, n}$ and $x_{n-1}=a_{n-1, n+1}\left(1-x_{n}\right)$ because $a_{n-1, n+1}=a_{n-1, n}$.

With the CADNA library if a particular combination of random roundings makes the $N$ elements of $x_{n}$ equal, then the round-off error on $x_{n}$ has vanished and the resulting $x_{n-1}, x_{n-2}, \ldots, x_{1}$ are false values and CADNA does not detect the failure.

Table 2.7 presents the percentages of failures with respect to the dimension $n$ and the number of samples $N$.

Tables 2.6 and 2.7 show that for computations in which only one rounding error is the dominant contribution to the final error, $N$ must be greater than 3 so that there is no failure. Then the choice of $N=3$ has to be explained. Indeed this is because for normal computing, several rounding errors contribute to the final error. The CADNA library uses $N=3$ and a probability of $95 \%$ for estimating the number of significant decimal digits. However it has been shown that if the user accepts an error of one unit in the number of significant decimal digits, then the probability of estimating it up to 1 is $99.94 \%$.

\subsection{Conclusion}

In this chapter the CESTAC method, which is a stochastic method for estimating the error propagation from both the FP arithmetic and the uncertainties of data issuing from sensors, has been presented. With this method the number (up to one) of significant digits on a computed numerical result can be evaluated. However this type of method was incorrectly implemented by S.G. Popovitch with his Prosolver software. In this software the $N$ runs are not synchronized and thus the control of numerical anomalies cannot be performed at the level of each elementary operators. Thus many numerical instabilities will not be not detected. It is for this reason that examples to expose the weakness of this software were proposed in [29] and [30]. Later using the ideas developed for the CESTAC method, the Monte Carlo method and the software Wonglediff were also proposed in [31-33] with the same drawbacks as those of Prosolver.

Indeed to be effective, the stochastic method requires that an eventual anomaly or instability is checked at the level of each elementary operation, i.e., an arithmetic operation, an order relation, or a branching. This requires that the $N$ samples representing the result of an operation are obtained synchronously and not by running the same code $N$ times in sequence. In other words, these methods are reliable if and only if they are implemented in the scope of granular computing and follow the model of DSA.

The theory of stochastic arithmetic, which is proposed in this chapter, provides a model for computation on approximate data. In this sense it aims at the same target as interval arithmetic except that the operands and operators are different. In the scope of granular computing the granules of stochastic arithmetic are independent Gaussian variables and the tools are the classical operators on Gaussian functions. These operators induce many algebraic structures and some of them have been presented.

The theory of DSA provides a model in which granules are composed of an $N$-tuple of $N$ samples of the same mathematical result of an arithmetical operator implemented in FP arithmetic. These samples differ from each other because the data are imprecise and because of different rounding. The operator working on these granules is an FP operator corresponding to the exact arithmetical operator which is performed $N$ times in a synchronous way with random rounding. Thus the result is also a granule. This granule is called a discrete stochastic number. It has been shown that the DSA operating on discrete stochastic numbers has many properties (but not all) of real numbers; in particular, the notion of stochastic zero has been defined. 
The CADNA library implements DSA and is able during the run of a code to analyze the effect of uncertainties of the data and of round-off error propagation on the result of each arithmetical operation. Thus any anomaly can be detected at this level. When such an anomaly is detected, a warning is written in a special file provided for the user. Hence, because of its correct implementation in the scope of granular computing the CADNA library does not fail when tested with the previously cited examples. This library has been successfully used for solving many problems belonging to the three categories of numerical methods.

In the field of linear algebra it has been used for the solution of linear systems using Gaussian elimination, GMRES [40], Orthomin(k) [41], and CGS [39] algorithms. It has enabled the optimization of collocation algorithms [27] and quadrature algorithms [42, 43]. It has also been used for checking the reliability of numerical methods in most fields of research in applied numerical mathematics: geology [44, 45], acoustics [46], solid mechanics [47], engine combustion [48], and atomic physics [49]. In all cases the CADNA library has always been successful.

Moreover, many future developments and applications of the CESTAC method, DSA, and CADNA are now possible particularly in the production of self-validated libraries requiring no programming effort in every domain of numerical analysis.

\section{References}

[1] J.G. Rokne. Interval arithmetic and interval analysis: an introduction. In: Granular Computing: An Emerging Paradigm. Physica-Verlag GmbH, Heidelberg, Germany, 2001, pp. 1-22.

[2] J.M. Chesneaux. Study of the computing accuracy by using a probabilistic approach. In: C. Ullrich (ed.), Contributions to Computer Arithmetic and Self-validating Methods, IMACS, NJ, 1990, pp. 19-30.

[3] J.M. Chesneaux. Etude théorique et implémentation en ADA de la méthode CESTAC. Thesis. Paris VI University, Paris, 1988.

[4] R.W. Hamming. On the distribution of numbers. Bell Syst. Tech. J. 49 (1970) 1609-1625.

[5] D. Knuth, The Art of Computer Programming 2. Addison-Wesley, Reading, MA, 1969.

[6] A. Feldstein and R. Goodman. Convergence estimates for the distribution of trailing digits. J. ACM. 23 (1976) 287-297.

[7] M. La Porte and J. Vignes. Evaluation statistique des erreurs numériques sur ordinateur. Proc. Canadian Comp. Conf. (1972) 414201-414213.

[8] M. La Porte and J. Vignes. Etude statistique des erreurs dans l'arithmétique des ordinateurs, application au contrôle des résultats d'algorithmes numériques. Numer. Math. 23 (1974) 63-72.

[9] M. La Porte and J. Vignes. Méthode numérique de détection de la singularité d'une matrice. Numer. Math. 23 (1974) 73-82.

[10] M. La Porte and J. Vignes. Evaluation de l'incertitude sur la solution d'un système linéaire. Numer. Math. 24 (1975) 39-47.

[11] J. Vignes and M. La Porte. Error analysis in computing. In: Information Processing 74, North-Holland, Amsterdam, 1974, pp. 610-614.

[12] P. Bois, and J. Vignes. An algorithm for automatic round-off error analysis in discrete linear transforms. Intern. J. Comput. Math. 12 (1982) 161-171.

[13] J.M. Chesneaux and J. Vignes. Sur la robustesse de la méthode CESTAC. C.R. Acad. Sci. Paris 307 (1988) $855-860$.

[14] J. Vignes. New methods for evaluating the validity of the results of mathematical computations. Math. Comput. Simul. 20 (4) (1978) 227-248.

[15] J. Vignes. Zéro mathématique et zéro informatique. C.A. Acad. Sci., Paris 303 (1) (1986) 997-1000; La Vie des Sciences, 4, (1) (1987) 1-13.

[16] J. Vignes. Discrete stochastic arithmetic for validating results of numerical software. Numer. Algoriths 37 (2004) 377-390.

[17] J. Vignes. A stochastic arithmetic for reliable scientific computation. Math. Comput. Simul. 35 (1993) 233-261.

[18] J. Vignes. Review on stochastic approach to round-off error analysis and its applications. Math. Comput. Simul. 30 (6) (1988) 481-491.

[19] J. Vignes and R. Alt. An efficient stochastic method for round-off error analysis, In: Accurate Scientific Computations, L.N.C.S 235, Springer-Verlag, New York, 1985, pp. 183-205.

[20] J.M. Chesneaux. L'Arithmétique stochastique et le logiciel CADNA. Habilitation à diriger les recherches. Université Pierre et Marie Curie, Paris, 1995. 
[21] J.M. Chesneaux and J. Vignes. Les fondements de l'arithmétique stochastique. C.R. Acad. Sci., Paris 315 (1992) $1435-1440$.

[22] J.M. Chesneaux. The equality relation in scientific computing. Numer. Algorithms 7 (1994) 129-143.

[23] R. Alt, and S. Markov. On the algebraic properties of stochastic arithmetic, comparison to interval arithmetic. In: W. Kraemer and J. Wolff von Gudenberg (eds), Scientific Computing, Validated Numerics, Interval Methods. Kluwer, Dordrecht, 2001, pp. 331-341.

[24] R. Alt, J.L. Lamotte and S. Markov. On the numerical solution to linear problems using stochastic arithmetic. In: Proceedings of the 2006 ACM Symposium on Applied Computing, Dijon France, (2006), pp. 1655-1659.

[25] S. Markov, R. Alt, and J.-L. Lamotte. Stochastic arithmetic: S-spaces and some applications, Numer. Algorithms 37 (1-4) (2004) 275-284.

[26] S. Markov and R. Alt. Stochastic arithmetic: addition and multiplication by scalars. Appl. Numer. Math. 50 (2004) 475-488.

[27] R. Alt and J. Vignes. Validation of results of collocation methods for ODEs with the CADNA library. Appl. Numer. Math. 20 (1996) 1-21.

[28] S.G. Popovitch. Prosolver, La Commande Electronique, France. Ashton-Tate, Torrance, CA, 1987.

[29] W. Kahan. The improbability of probabilistic error analyses. In: UCB Statistics Colloquium. Evans Hall, University of California, Berkeley, 1996. http://www.ca.berkeley.edu/wkahan/improber.ps.

[30] W. Kahan. How futile are mindless assessments of round-off in floating point computation. Householder Symposium XVI, 2005, http://www.cs.berkeley.edu/wkahan/Mindless.pdf.

[31] D.S. Parker. Monte Carlo Arithmetic: Exploiting Randomness in Floating Point Arithmetic. Report of computer science department, UCLA, Los Angeles, March, 30, 1997.

[32] D.S. Parker, B. Pierce, and D.R. Eggert. Monte Carlo arithmetic: how to gamble with floating point and win. Comput. Sci. Eng. (2000) 58-68.

[33] P.R. Eggert, and D.S. Parker. Perturbing and evaluating numerical programs without recompilation - the wonglediff way. Softw. Pract. Exp. 35 (2005) 313-322.

[34] J. Vignes. A stochastic approach to the analysis of round-off error propagation: a survey of the CESTAC method. In: Proceedings of the 2nd Real Numbers and Computer Conference, Marseille, France, 1996, pp. 233-251.

[35] M. Pichat and J. Vignes. Ingénierie du contrôle de la précision des calculs sur ordinateur. Technip, Paris (1993).

[36] Cadna user's guide, http://www.lip6.fr/cadna.

[37] M. Daumas, and J.M. Muller. Qualité des Calculs sur Ordinateur. Masson, Paris, 1997.

[38] J.R. Westlake. Handbook of Numerical Matrix Inversion and Solution of Linear Equations. Wiley. New York, 1968.

[39] J.M. Chesneaux, and A. Matos. Breakdown and near breakdown control in the CGS algorithm using stochastic arithmetic. Numer. Algorithms 11 (1996) 99-116.

[40] F. Toutounian. The use of the CADNA library for validating the numerical results of the hybrid GMRES algorithm. Appl. Numer. Math. 23 (1997) 275-289.

[41] F. Toutounian. The stable $A^{T} A$-orthogonal $s$-step $\operatorname{Orthomin}(k)$ algorithm with the CADNA library. Numer. Algorithms 17 (1998) 105-119.

[42] F. Jézéquel and J.M. Chesneaux. Computation of an infinite integral using Romberg's method. Numer. Algorithms 36 (2004) 265-283.

[43] F. Jézéquel. Dynamical control of converging sequences computation. Appl. Numer. Math. 50 (2004) 147-164.

[44] F. Delay and J.-L. Lamotte. Numerical simulations of geological reservoirs: improving their conditioning through the use of entropy. Math. Compute. Simul. 52 (2000) 311-320.

[45] J.-L. Lamotte and F. Delay. On the stability of the 2D interpolation algorithms with uncertain data. Math. Comput. Simul. 43 (1997) 183-190.

[46] J.M. Chesneaux, and A. Wirgin. Reflection from a corrugated surface revisited. J. Acoust. Soc. 96 (2 pt. 1) (1993) $1116-1129$.

[47] N.C. Albertsen, J.-M. Chesneaux, S. Christiansen, and A. Wirgin. Evaluation of round-off error by interval and stochastic arithmetic methods in a numerical application of the Rayleigh theory to the study of scattering from an uneven boundary. In: G. Cohen (ed), Proceedings of the Third International Conference on the Mathematical and Numerical Aspects of Wave Propagation, SIAM, Philadelphia, 1995, pp. 338-346.

[48] S. Guilain, and J. Vignes. Validation of numerical software results. Application to the computation of apparent heat release in direct injection diesel engine. Math. Comput. Simul. 37 (1994) 73-92.

[49] N.S. Scott, F. Jézéquel, C. Denis, and J.M. Chesneaux. Numerical 'health check' for scientific codes: the CADNA approach. Comput. Phys. Commun. 176 (8) (2007) 507-521. 